Results This meta-analysis includes 18 studies encompassing data on 13.755 very preterm/VLBW infants. Very preterm/VLBW infants with perinatal infections have poorer mental $(-0.25 \mathrm{SD}, p<0.001)$ and motor development $(-0.37 \mathrm{SD}, p<0.001)$ compared to very preterm/VLBW infants without infections. Mental development is most impaired by necrotizing enterocolitis (NEC, -0.40 SD $p<0.001$ ) and meningitis $(-0.37 \mathrm{SD} p<0.001)$. Motor development is most impaired by NEC $(-0.66 \mathrm{SD} p<0.001)$. Chorioamnionitis did not affect mental or motor development $(-0.05 \mathrm{SD}, p=0.37$ and $0.19 \mathrm{SD}$, $p=0.082$ ).

Conclusions Postnatal infections have detrimental effects on mental and motor development in very preterm/VLBW infants. This effect adds up to the well-known detrimental effect of prematurity and highlights the importance of infection prevention in these vulnerable infants.

\section{COMPARISON OF REGIONAL CEREBRAL BLOOD FLOW DISTRIBUTION AFTER AEROSOLIZED VERSUS INSTILLED SURFACTANT IN PRETERM LAMBS}

doi:10.1136/archdischild-2012-302724.0411

'VE Mielgo, 'C Rey-Santano, 'E Ruizdel-Yerro, ${ }^{2} \mathrm{~A}$ Vallsi-Soler, ${ }^{1} \mathrm{X}$ Murgia. ' $R e s e a r c h$ Unit; ${ }^{2}$ Neonatal Intensive Care Unit, Cruces University Hospital, Baracaldo, Spain

Background Rapid Intratracheal instillation of surfactant (SF) in preterm neonates with respiratory distress syndrome (RDS) has been associated with cerebral haemodynamic disturbances. Aerosolized surfactant might potentially avoid these disturbances through a gradual improvement of lung function.

Objetive To compare carotid blood flow (CBF) and regional cerebral blood flow (RCBF) distribution in preterm lambs after treatment with instilled or aerosolized surfactant.

Methods 12 preterm lambs (133d GA) were randomized to receive instilled (SF-Bolus, $\mathrm{n}=6$ ) or aerosolized (SF-Aero, $\mathrm{n}=6$ ) surfactant. CBF was measured at foetal life, baseline, 5, 15 and 30 minutes after the initiation of surfactant therapy and thereafter, every 30 minutes until the end of the experiment. RCBF was determined using coloured microspheres technique at foetal life and 5, 60, 180 and 360 minutes after the start of surfactant therapy. Brain samples of striatum, thalamus and hippocampus grouped as inner zones, cortical zones and cerebellum and brain-stem (CB-B) were analyzed. ANOVA, $p<0.05$.

Results Following SF-Bolus administration a marked increase in CBF was observed for the first $30 \mathrm{~min}$ in comparison to SF-Aero group. 1 hour after treatment, however, both groups had similar $\mathrm{CBF}$ values. Immediately after SF instillation and during the ventilatory support, RCBF in inner (thalamus and hippcampus) and in $\mathrm{CB}-\mathrm{B}$ zones was increased in SF-bolus group in comparison with SF-Aero group. No significant differences were detected in cortical blood flow.

Conclusion In preterm lambs with RDS, aerosolized surfactant produced a different cerebral haemodynamic pattern than did SF bolus instillation. These observations should be carefully explored (FIS10/00943)

\section{COMBINATION OF GENOMIC TECHNOLOGIES AND CONSANGUINITY IN ORDER TO IDENTIFY PATHOGENIC VARIANTS IN RECESSIVE DISORDERS}

doi:10.1136/archdischild-2012-302724.0412

'P Makrythanasis, 'M Nelis, 'FA Santoni, ${ }^{2} \mathrm{M}$ Guipponi, ${ }^{2} \mathrm{~F}$ Béna, ${ }^{1} \mathrm{~A}$ Vanier, ${ }^{2} \mathrm{G}$ DuriauxSail, ${ }^{2} \mathrm{~S}$ Gimelli, ${ }^{2} \mathrm{E}$ Stathaki, ${ }^{1} \mathrm{E}$ Falconnet, ${ }^{3} \mathrm{~S}$ Temtamy, ${ }^{4} \mathrm{~A}$ Megarbane, ${ }^{3} \mathrm{M}$ Aglan, ${ }^{3} \mathrm{M}$ Zaki, ${ }^{2} \mathrm{~S}$ Fokstuen, ${ }^{2} \mathrm{~A}$ Bottani, ${ }^{5} \mathrm{~A}$ Masri, ${ }^{6} \mathrm{~S}$ Psoni, ${ }^{6} \mathrm{~S}$ Kitsiou, ${ }^{6} \mathrm{H}$ Frissyra, ${ }^{6} \mathrm{E}$ Kanavakis, ${ }^{7} \mathrm{~N}$ All-Allawi, ${ }^{8} \mathrm{~A}$ Sefiani, ${ }^{9} \mathrm{~S}$ Al-Hait, ${ }^{8}$ S Elalaoui, ${ }^{4} \mathrm{~N}$ Jalkh, ${ }^{10,11}$ L Al-Gazali, ${ }^{10,11} \mathrm{~F}$ Al-Jasmi, ${ }^{12} \mathrm{H}$ Chaabouni Bouhamed, ${ }^{1} \mathrm{H}$ Hamamy, ${ }^{1}{ }^{2} \mathrm{SE}$ Antonarakis. ${ }^{1}$ Department of Genetic Medicine and Development, University of Geneva; ' ${ }^{2}$ Service of Genetic Medicine, University Hospitals of Geneva, Geneva, Switzerland; ${ }^{3}$ Department of Clinical Genetics, National
Research Cenrte Cairo, Cairo, Egypt; "Medical Genetics Unit, Saint Joseph University, Beirut, Lebanon; ${ }^{5}$ Pediatric Department, Professor, Amman, Jordan; ${ }^{6}$ Department of Medical Genetics, University of Athens, Athens, Greece; 'Department of Pathology, College of Medicine, University of Dohuk, Dohuk, Iraq; ${ }^{8}$ Département de génétique médicale, Institut National d'Hygiène, Rabat, Morocco, ${ }^{9}$ Genetic Clinic, Al Amal Maternity Hospital, Amman, Jordan; ${ }^{10}$ Department of Paediatrics, Faculty of Medicine and Health Sciences, United Arab Emirates University; " Department of Pediatrics, Tawam Hospital, United Arab Emirates University, Al-Ain, United Arab Emirates; ${ }^{12}$ Department of Human Genetics, University Tunis El Manar, Faculty of Medicine, Tunis, Tunisia

Consanguinity and inbreeding increase the sharing of alleles among individuals; thus a considerable number of autosomal recessive phenotypes occur in offspring(s) of consanguineous couples. We have collected samples from consanguineous families with different phenotypes of unknown etiology that are compatible with autosomal recessive transmission, in order to identify the responsible functional genomic variation. 42 families of different ethnic background have been collected so far. From each family, DNA from the patient(s), unaffected siblings and the parents is extracted. Samples are i/analyzed by array-CGH for the detection of homozygous deletions; ii/genotyped with a 720K SNP-array in order to identify Runs of Homozygosity and the areas of the genome that could include the causative variant; iii/exome sequenced (one affected individual/ family). Mean coverage is $130 \mathrm{x}$ and $98.2 \%$ of the coding region of RefSeq is covered at least $8 x$. By comparing the genotyping and sequencing data, we found that Single Nucleotide Variants (that passed the quality threshold) were detected with a specificity of $99.95 \%$, sensitivity of $97.7 \%$, Positive Predictive Value $99.2 \%$ and Negative Predictive Value 98.6\%. On average we identified 21901 variants/exome. So far we analysed 26 families and identified the causative variation in known genes in 3 of them:VLDLR, FKTN and DMP1. In 12 families 23 candidate genes/variants have been identified(more than 1 candidate genes/family). In 11 families the likely molecular defect has not been identified. Consanguineous families provide an opportunity to identify pathogenic variants responsible for recessive phenotypes and rapidly fill in the gap between genotype and phenotype.

\section{Poster Symposium Presentations - Nursing}

\section{EFFECTIVENES OF THE CALA-DOL, A NEW NON PHARMACOLOGICAL METHOD TO REDUCE PAIN IN CHILDREN UNDERGOING INTRAMUSCULAR IMMUNIZATION: RANDOMIZED CONTROLLED TRIAL}

doi:10.1136/archdischild-2012-302724.0413

${ }^{1} \mathrm{M}$ Calamini, ${ }^{2} \mathrm{C}$ Neri, ${ }^{1} \mathrm{~F}$ Festini. 'University of Florence; ${ }^{2}$ Meyer Children Hospital, Florence, Italy

Background and Aims Procedural pain due to immunization is often untreated due to the limited effectiveness of available methods. The aim of this study is to value the effectiveness of a psychosomatic technique called Cala-Dol for the reduction of pain caused by immunization in children.

Materials and methods: Randomized controlled trial. 500 children aged 3 to 13 undergoing intramuscular vaccination against meningitis in the deltoid muscle were enrolled and randomized into two groups. Group A received standard care (no intervention), group $\mathrm{B}$ received the Cala-Dol technique. Cala-Dol consists in a combination of local cutaneous stimulation with two small rubber balls -one smooth and one with soft protrusions- and distraction. The cost of the devices is 2 Euros. Two Pain was measured with a 0-10 faces Wong scale (up to 7 years) or a 0-10 Visual analog scale (VAS).

Results Mean pain score in Group B (2.2, sd 1.89) was significantly lower than pain score in Group A (3.38, sd 1.84, p=0.0001). Children were stratified according to age. With regards to age, pain score 